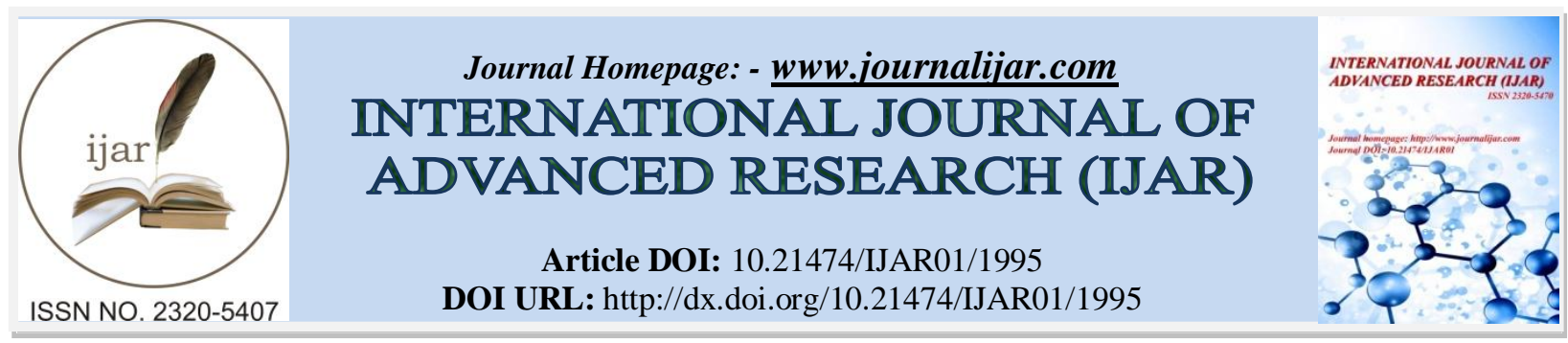

RESEARCH ARTICLE

\title{
CHOIX DE L'AUDITEUR EXTERNE : QUELLES PRATIQUES POUR LES ENTREPRISES
} TUNISIENNES?

Azhaar Lajmi, Hasna Chaibi and Abir Ayari.

\section{Manuscript Info}

Manuscript History

Received: 19 August 2016

Final Accepted: 22 September 2016

Published: October 2016

Key words:-

organizational complexity, leverage,

investment opportunities, audit

committee, audit quality.

\begin{abstract}
This study aims to examine the behavior of Tunisian companies in their choice of the quality of external auditor, basing on some organizational and governance characteristics. Our logistic regression is estimated on the basis of a sample of panel data composed by 25 Tunisian companies listed on the Tunisian Stock Exchange, during the period 2007-2012. We show a complementary relationship between the size of Tunisian companies, the investment opportunity and the external audit quality. However, we find a negative relationship between direct labor, debt and the choice of a better audit quality.
\end{abstract}

Copy Right, IJAR, 2016,. All rights reserved.

\section{Introduction :-}

Durant la dernière décennie, nous avons assisté à un accroissement d'états financiers manipulés et une augmentation du nombre de faillites de certaines sociétés cotées, lesquels ont été à l'origine d'une véritable crise de confiance sur le plan international. La disparition d'un cabinet d'audit, qui a été à un moment donné, une des entités de renommée composant le réseau international Big, pose le problème du choix du cabinet d'audit comme étant un des mécanismes de gouvernance, permettant l'instauration d'une surveillance accrue. Il est devenu, par conséquent, essentiel suite aux affaires qui ont bouleversé les milieux économiques de redonner confiance aux différentes parties prenantes lésées par ces nombreux scandales financiers, en mettant en place des réformes fondamentales dans la gouvernance d'entreprise. À cet effet, les institutions régulatrices adoptent de plus en plus des lois sur la sécurité financière. Elles permettent essentiellement de renforcer les règles de bonne gouvernance et d'améliorer la qualité de l'information financière ainsi que la qualité de l'audit externe. Parmi ces lois, nous pouvons citer la loi SarbanesOxley (SOX) de 2002 aux États-Unis, la loi sur la sécurité financière de 2003 en France, le code Lippens 2005 en Belgique et la loi relative au renforcement de la sécurité financière de 2005 en Tunisie.

Dans ce cadre, le présent article vise à déterminer certains facteurs affectant la demande de l'audit externe de meilleure qualité des entreprises tunisiennes. Les offres sur le marché d'audit sont divisées en deux groupes : (1) les grands cabinets d'audit qui sont supposés offrir une bonne qualité de service d'audit grâce à l'utilisation de la technologie avancée (Craswell et al., 2002) et (2) les autres cabinets nationaux qui sont supposés offrir un service d'audit de moins bonne qualité. Cette diversification de la qualité de l'audit nous a motivé focaliser notre attention sur les déterminants du choix des auditeurs externes pour les entreprises tunisiennes approché par l'appartenance ou non aux grands cabinets d'audit (Big4). En effet, ces réseaux sont considérés comme les plus efficaces sur le marché d'audit, dans la mesure où ils sont caractérisés par une forte réputation et par conséquent, ils sont les plus susceptibles de produire une meilleure qualité de service.

En ce sens, plusieurs caractéristiques de l'entreprise et de gouvernance sont susceptibles d'affecter le choix de l'audit externe. C'est sous ce même angle que nous analysons, dans cet article, l'impact de la complexité 
organisationnelle, de l'endettement, de la participation des investisseurs institutionnels dans le capital de la société, des opportunités d'investissement et de l'indépendance du comité d'audit sur le choix des auditeurs externes des entreprises tunisiennes. Le lien entre ces variables s'appuie sur la théorie de l'agence (Jensen et Meckling, 1976). Ces différentes relations ont suscité notre intérêt en raison de l'importance des enjeux économiques pour les entreprises des pays émergents, notamment la Tunisie, étant donné que la qualité de l'audit externe représente la pierre angulaire de la divulgation d'une information financière de qualité. De plus, le contexte tunisien nous a semblé un terrain approprié pour une telle recherche dans le domaine de la comptabilité. En effet, l'intérêt central porté par les institutions régulatrices tunisiennes quant au renforcement des règles de bonne gouvernance, nous a poussés à étudier ce sujet dans un tel contexte. En Tunisie, le législateur a introduit à travers la loi n ${ }^{\circ} 2005-96$ du 18 octobre 2005 relative au renforcement de la sécurité des relations financières une mesure concernant l'indépendance des auditeurs, ce qui prouve que le législateur tunisien a une volonté très forte de développer la compétence des commissaires aux comptes et de renforcer leur indépendance. En outre, nous avons constaté que la plupart des études antérieures s'est intéressée à examiner la relation entre certaines mécanismes internes de gouvernance et le choix de l'auditeur. Nous voulons étendre cette lignée d'études passées et ajouter la complexité organisationnelle et les opportunités d'investissement comme facteurs explicatifs de la qualité de l'audit externe.

Notre contribution théorique consiste à enrichir la littérature relative à la relation entre la gouvernance et la qualité de l'audit externe. Notre apport est d'autant plus visible étant donné que les recherches antérieures se sont intéressées seulement à quelques aspects de la gouvernance aussi bien dans le contexte anglo-saxon qu'européens. Quant à notre contribution empirique et méthodologique, celle-ci consiste en l'utilisation de la méthodologie de données de panel et en la multiplication des tests de robustesse.

Le présent article est structuré de la façon suivante. La partie suivante décrit le cadre théorique traitant de la relation entre les caractéristiques de l'entreprise et le choix de l'audit externe ainsi que la formulation des hypothèses de recherche. La troisième partie expose notre méthodologie de travail. Les résultats et les interprétations font l'objet de la partie 4 , suivie de la partie 5 présentant les tests de sensibilité. Enfin, la dernière partie permet de conclure.

\section{Caractéristiques de l'entreprise et choix de l'auditeur externe: fondement théorique et développement des hypothèses :- \\ Complexité organisationnelle et choix de l'auditeur externe :- \\ Un cabinet d'audit d'envergure international a accès à plus de moyens matériels et humains ainsi qu'à une meilleure expertise pour gérer la complexité d'une mission d'audit menée dans des entreprises présentant une complexité de ses opérations.}

Les recherches traitant de la relation entre la complexité de l'organisation et la qualité de l'audit externe présentent des résultats controverses. Hay et Davis (2004), Omri et Dallai (2013) et Hsu et al. (2015) confirment que les firmes les plus complexes préfèrent choisir un audit externe de meilleure qualité. Selon ce point de vue, de telles entreprises devraient choisir un auditeur de meilleur qualité, utilisant une meilleure technologie et possédant des compétences nécessaires pour répondre aux besoins en temps opportun et pour bien gérer une entité et par conséquent un audit complexe. Nekhili et al. (2009) considèrent que la diversification sectorielle renvoie au degré de la complexité organisationnelle d'une firme. Le résultat de leur étude montre, toutefois, l'absence de relation significative entre la diversité sectorielle et le choix de l'auditeur. La première hypothèse que nous nous proposons de tester s'énonce comme suit :

Hypothèse 1: La complexité de l'organisation de la firme cliente a un effet positif sur le choix des auditeurs externes.

\section{Endettement de la firme et choix de l'auditeur externe :-}

Vu sous l'angle de la théorie de l'agence, l'endettement peut être considéré comme une solution aux conflits actionnaires-dirigeants et ce, par le caractère contractuel de cette source de financement. L'auditeur intervient justement pour asserter le respect des clauses du contrat. Le rôle de l'auditeur prend, dès lors, de plus en plus d'importance au fur et à mesure que l'endettement augmente. Il en est de même lorsque des conflits surgissent entre actionnaires et créanciers. D'ailleurs, Joshi et Al-Bastaki (2000) montrent que les firmes les plus endettées payent des honoraires d'audit plus élevés. En conséquence, la qualité de l'audit semble être comme un dispositif de surveillance approprié pour régler les conflits d'agence entre les actionnaires et les créanciers. Toutefois, Kane et 
Velury (2005) dans le contexte américain, Dumontier et al. (2006) dans le contexte tunisien et Lajmi et Gana (2013a) dans le contexte belge trouvent qu'il n'existe aucune relation entre l'endettement et le choix de l'auditeur externe de meilleure qualité. Par conséquent, nous avançons l'hypothèse ci-après :

Hypothèse 2 : L'endettement de la firme a un effet positif sur le choix des auditeurs externes.

\section{Propriété institutionnelle et choix de l'auditeur externe :-}

La propriété institutionnelle influence les relations d'agence au sein de la firme dans la mesure où elle joue un rôle de contrôle (Jensen et Meckling, 1976). Dans ce sens, ces derniers affirment que les investisseurs institutionnels, en vertu de leurs participations importantes, tendent à être plus actifs dans la gouvernance de la firme et bénéficient potentiellement d'un plus grand pouvoir de contrôle et de gestion. Lajmi et Gana (2012) supposent une relation positive entre la propriété institutionnelle et la qualité de l'audit. Sur le plan théorique, les auteurs soutiennent la thèse de complémentarité entre ces deux mécanismes de contrôle, puisque de tels actionnaires, considérés comme de bons contrôleurs et possédant des parts importantes dans le capital de l'entreprise, veulent assurer plus de contrôle pour protéger leurs intérêts en demandant un audit de meilleure qualité.

Sur le plan empirique, Abdullah (2008) montre que les sociétés ont tendance à faire appel à des auditeurs de type «Big», à mesure que le niveau de la propriété institutionnelle augmente, et ce afin, d'améliorer et de renforcer le contrôle de l'entreprise. A contrario, d'autres études, notamment celles de Azibi et al. (2011) dans le contexte français et Lajmi et Gana (2012) dans le contexte belge, montrent une relation plutôt négative entre ces deux concepts.

Par ailleurs, nous formulons notre troisième hypothèse comme suit :

Hypothèse 3 : La part détenue par les investisseurs institutionnels dans le capital de la société a un effet positif sur le choix de l'auditeur externe.

Opportunité d'investissement et choix de l'auditeur externe :-

Jensen et Meckling (1976) indiquent que l'audit est un moyen utilisé par les dirigeants pour assurer aux propriétaires qu'ils ne se comportent pas de façon opportuniste. Cet argument motive pour un choix d'audit de meilleure qualité.

Toutefois, la littérature financière n'indique pas qu'un cabinet de type « Big » pourrait être également utile pour les entreprises ayant des opportunités d'investissement élevés (Lai, 2009). En effet, trop peu d'études traitent de la relation entre les opportunités d'investissement et la qualité de l'audit externe. Saghafi et Motamedi (2011) examinent la relation entre la qualité de l'audit et l'efficacité des investissements dans les sociétés ayant des opportunités d'investissement élevées. Les résultats montrent que, si dans une société, le niveau des opportunités d'investissement est élevé, celles-ci auront tendance à engager des auditeurs de meilleure qualité. Ceci est de nature à élever l'efficacité des investissements. Lai (2009) montrent également que, dans les sociétés qui ont de fortes opportunités d'investissement, la probabilité de choisir un auditeur externe de meilleure qualité est élevée. Ce même résultat a été prouvé par l'étude de Omri et Dallai (2013). Nous énonçons, par ailleurs, l'hypothèse suivante :

Hypothèse 4: Le choix d'auditeurs externes de meilleure qualité augmente avec le niveau des opportunités d'investissement qui se présente à l'entreprise.

\section{Indépendance du comité d'audit et choix de l'auditeur externe :-}

En se basant sur la théorie de l'agence, Fama et Jensen (1983) avancent que les administrateurs indépendants qui constituent le comité d'audit sont susceptibles de contrôler les dirigeants et les administrateurs internes. En outre, la théorie de l'agence stipule que les administrateurs indépendants sont de nature à limiter les conflits d'agence entre les dirigeants et les actionnaires. Ainsi, l'indépendance du comité d'audit améliore son efficacité (Azibi, 2014). Ce raisonnement soutient l'idée que de tels administrateurs sont considérés comme des contrôleurs efficaces. Leurs décisions seront donc plus objectives que celles prises par les administrateurs internes. Ils orientent, également, le choix des auditeurs vers des contrôleurs compétents, afin d'avoir des états financiers plus crédibles. De plus, ils demandent une assurance additionnelle de l'auditeur, afin de protéger leur réputation. Broye (2009) étudie la relation entre les caractéristiques du comité d'audit et la qualité de l'audit externe approchée par les honoraires d'audit. Les résultats montrent que cette relation est positive. Dans une recherche plus récente, Lajmi et Gana (2013b) montrent 
un effet positif et statistiquement significatif de l'indépendance du comité d'audit sur la demande d'une meilleure qualité d'audit externe mesurée par un indice composite de plusieurs attributs de la qualité de l'audit.

Ces résultats relativement concordants nous amènent à poser l'hypothèse suivante:

Hypothèse 5 : Le choix de l'auditeur externe est positivement affecté par l'indépendance du comité d'audit.

\section{Méthodologie empirique :-}

A présent, nous décrivons l'échantillon ainsi que le modèle et les variables de notre étude.

Présentation de l'échantillon de l'étude :-

Notre échantillon, observé sur une période allant de 2007 à 2012, est composé de 25 sociétés cotées à la Bourse des Valeurs Mobilières de Tunis (BVMT) réparties en six secteurs d'activité à savoir : l'industrie, le commerce, les services, l'agroalimentaire, l'immobilier et la communication. Les banques, les assurances et les autres institutions financières ne sont pas inclues dans l'échantillon, compte tenu de la spécificité des règles relatives à la préparation de leurs états financiers et ce, afin d'assurer l'homogénéité des observations.

Mesure de la variable dépendante : qualité de l'audit externe :-

D'après la littérature comptable, la réputation du cabinet d'audit est la mesure la plus utilisée pour évaluer sa qualité. En effet, Palmorse (1988) montre que les cabinets d'audit ayant une bonne réputation sont ceux qui ont moins de litiges et qui ne sont pas cités dans les scandales financiers et les affaires frauduleuses. Ainsi, les grands cabinets d'audit présentent une meilleure réputation, en étant indépendant, et fournissent de prestations de meilleure qualité par rapport aux autres cabinets « Non Big» (Miko et Kamardin, 2015). Ces derniers ajoutent que les « Big 4 » ont la capacité de fournir une qualité d'audit plus élevée pour les raisons suivantes : ils ont de nombreux clients, de ressources importantes, de la technologie et du personnel hautement qualifié pour le travail d'audit. Dans le cadre de notre travail, nous optons pour cette mesure de la qualité de l'audit externe. Il s'agit d'une variable dichotomique qui prend la valeur de 1 si l'entreprise est auditée par un «Big 4 », 0 sinon.

\section{Définitions des variables explicatives :-}

Dans ce qui suit, nous présentons les variables d'intérêt et de contrôle.

La complexité organisationnelle :-

Tout au long de cette recherche, nous utilisons deux mesures de la complexité de l'organisation de la firme cliente. MOD=COI= Rémunération du personnel / Total des dépenses d'exploitation

Ce ratio (COI) reflète la complexité opérationnelle et informationnelle d'une organisation (Hay et Davis, 2004), avec MOD est la main d'œuvre directe.

$\mathrm{CTR}=($ Stocks + Créances + Produits à recevoir $) /$ Total des actifs

Ce ratio (CTR) reflète la complexité transactionnelle (Hay et al., 2006).

Plus l'entreprise possède un grand nombre de transactions, plus la probabilité d'erreurs dans ses états financiers est élevée, ce qui explique le recours à un audit externe de meilleure qualité permettant la détection des anomalies.

En ce sens, dans une organisation complexe, le grand volume de transactions ainsi que la forte intensité de main d'œuvre font augmenter le risque d'erreurs et d'omissions. Par conséquent le recours à un auditeur externe de bonne qualité devient nécessaire.

L'endettement (END) :-

Nous suivons Lajmi et Gana (2013a,b) et Hsu et al. (2015) en mesurant l'endettement par le rapport entre les dettes à long terme et le total des actifs de l'entreprise.

La propriété institutionnelle (PINST) :-

La plupart des études montre une relation positive entre la participation des investisseurs institutionnels et le choix des auditeurs externes de meilleure qualité (Abdullah, 2008). Cette variable est calculée en fonction du nombre d'actions détenues par les institutionnels déflaté par le nombre total des actions en circulation de la firme. 
Les opportunités d'investissement :-

Nous optons pour la mesure basée sur le prix. Celle-ci se base sur l'idée que si les prévisions de croissance de la firme sont au moins partiellement prises en compte dans les prix des actions, alors les firmes en croissance devraient avoir des valeurs de marché élevées relativement à la valeur des actifs en place. Nous citons des exemples de ce type de mesure :

* $\quad$ Market to Book Equity (Gul et Tsui, 1998).

* Book to Market Value of Asset (Smith et Watts, 1992).

Nous désignons par MBA et MBE, les mesures des opportunités d'investissement des entreprises de notre étude. MBA (market to book asset) $=($ Valeur de marché des capitaux propres +Dettes à long terme)/total des actifs. Ce ratio est utilisé par Lang et Litzenberg (1989).

MBE (market to book equity)=Valeur de marché des capitaux propres/valeur comptable des capitaux propres.

L'indépendance du comité d'audit (INDCAUD) :-

Nous approchons l'indépendance du comité d'audit par le pourcentage de ses administrateurs indépendants (Lajmi et Gana, 2013b ; Miko et Kamardin, 2015).

Dans le but d'améliorer le pouvoir explicatif du modèle de notre étude, nous retenons les variables de contrôle suivantes :

La taille de l'entreprise (TAILLE) :-

Dumontier et al. (2006) et Lajmi et Gana (2013a,b) mesurent la taille de l'entreprise par le logarithme du total de l'actif. Dumontier et al. (2006) constatent que les efforts des auditeurs et la qualité de service d'audit augmentent en fonction de la taille de la firme.

La rentabilité de l'entreprise (ROA) :-

Selon la théorie de signal, la firme annonce sa qualité aux investisseurs quand sa performance est bonne. Il s'agit de la capacité de l'entreprise à générer de la rentabilité au départ de ses actifs. Lajmi et Gana (2012) indiquent que la rentabilité de l'entreprise est positivement liée à la qualité de l'audit. Cette variable à été aussi utilisée par Choi et Lee (2014). Cette rentabilité correspond au rendement de l'actif mesuré par le rapport entre le résultat avant impôts et intérêts et le total des actifs.

Spécification du modèle de la régression logistique :-

Notre régression est estimée sur la base d'un panel composé de 150 observations. Dans le but de tester nos hypothèses de recherche, nous régressons la qualité de l'audit externe en fonction de certaines caractéristiques de la firme, tout en contrôlant l'effet de la taille de l'entreprise et de sa rentabilité.

Par conséquent, notre premier modèle est formulé comme suit:-

Choix $_{i, t}=\propto_{0}+\propto_{1}$ MOD $_{i, t}+\propto_{2}$ END $_{i, t}{ }^{+} \propto_{3}$ PINST $_{i, t^{+}} \propto_{4}$ MBA $_{i, t^{+}} \propto_{5}$ INDCAUD $_{i, t}{ }^{+} \propto_{6}$

$\boldsymbol{R O} A_{i, t}+\propto_{7}$ TAILLE $E_{i, t}+\varepsilon_{i, t}$

Avec : Choix, MOD, END, PINST, MBA, INDCAUD, ROA et TAILLE correspondent aux variables précédemment définies.

$i$ représente les entreprises retenues dans notre échantillon et ;

$t$ concerne la période de l'étude.

$\propto_{1}, \propto_{2}, \propto_{3}, \propto_{4}, \propto_{5}, \propto_{6}$ et $\propto_{7}$ : représentent les coefficients à estimer du modèle.

$\varepsilon$ constitue le terme d'erreur.

Il est à noter que notre modèle de travail est inspiré de celui utilisé par Azibi et al. (2011) sur le marché français avec certaines différences au niveau des variables retenues.

\section{Analyse des résultats :-}

Dans ce qui suit, nous analyserons les résultats issus des statistiques descriptives. Par la suite, nous interpréterons les résultats empiriques de notre premier modèle. 


\section{Statistiques descriptives :-}

Nous présentons, le tableau 1 et le tableau 2 qui résument, respectivement, les résultats descriptifs des variables pour les entreprises auditées par un «Big4 » et par un «non Big4».

Tableau 1:- Statistiques descriptives des variables des firmes auditées par un « Big4» (2007-2012)

Les statistiques descriptives de notre échantillon des entreprises tunisiennes cotées auditées par un «Big4 » sont présentées au niveau du tableau 1. MOD est un attribut de la complexité opérationnelle et informationnelle. CTR représente la complexité transactionnelle. END présente le ratio d'endettement à long terme de la firme. PINST est la participation institutionnelle. MBA est le market to book asset, MBE est le market to book equity. INDCAUD est l'indépendance du comité d'audit. ROA mesure la rentabilité de la firme. TAILLE est la taille de la firme.

\begin{tabular}{|c|c|c|c|c|c|}
\hline & \multicolumn{5}{|c|}{ «Big4» } \\
\hline Variables & $\begin{array}{c}\text { Nombre } \\
\text { d'observations }\end{array}$ & Moyenne & Ecart type & Minimum & Maximum \\
\hline MOD & 49 & 0.1333469 & 0.0947898 & 0.039 & 0.409 \\
\hline CTR & 49 & 0.481898 & 0.1695991 & 0.168 & 0.765 \\
\hline END & 49 & 0.0850408 & 0.1032287 & 0 & 0.404 \\
\hline PINST & 49 & 24.78265 & 0.2100499 & 0 & 76.31 \\
\hline MBA & 49 & 1.412143 & 1.242171 & 0.074 & 6.405 \\
\hline MBE & 49 & 7.222939 & 7.598418 & 0.297 & 34.499 \\
\hline INDCAUD & 49 & 0.3690367 & 0.379215 & 0 & 1 \\
\hline ROA & 49 & 0.0572041 & 0.0919055 & -0.156 & 0.203 \\
\hline TAILLE & 49 & 18.43584 & 0.6474119 & 16.75 & 19.646 \\
\hline
\end{tabular}

En fonction des résultats issus des tableaux 1 et 2, nous remarquons que les firmes tunisiennes auditées par un «Big4 » ont en moyenne une MOD inférieure à celle des autres firmes qui sont auditées par les «non Big4 » $(13,33 \%<25,56 \%)$, ce qui montre que les firmes auditées par un «Big4» ont un nombre réduit de mains d'œuvre par rapport aux autres firmes. De même, nous constatons que la moyenne de la variable CTR pour les entreprises auditées par un «Big4» est supérieure à celles qui sont auditées par les «non Big4» $(48,19 \%>46,22 \%)$. Ceci montre que les entreprises qui sont auditées par un «Big4 » ont des opérations transactionnelles légèrement plus complexes que les autres firmes.

Tableau 2 :- Statistiques descriptives des variables des firmes auditées par un «non Big4» (2007-2012) Les statistiques descriptives de notre échantillon des entreprises tunisiennes cotées auditées par un «non Big4 » sont présentées au niveau du tableau 2. MOD est un attribut de la complexité opérationnelle et informationnelle. CTR représente la complexité transactionnelle. END présente le ratio d'endettement à long terme de la firme. PINST est la participation institutionnelle. MBA est le market to book asset, MBE est le market to book equity. INDCAUD est l'indépendance du comité d'audit. ROA mesure la rentabilité de la firme. TAILLE est la taille de la firme.

\begin{tabular}{|c|c|c|c|c|c|}
\hline & \multicolumn{5}{|c|}{ «Non big4» } \\
\hline Variables & $\begin{array}{c}\text { Nombre } \\
\text { d'observations }\end{array}$ & Moyenne & Ecart type & Minimum & Maximum \\
\hline MOD & 101 & 0.2556337 & 0.3068853 & 0.023 & 0.709 \\
\hline CTR & 101 & 0.4622277 & 0.2229573 & 0.019 & 0.853 \\
\hline END & 101 & 0.1007088 & 0.0981376 & 0 & 0.32 \\
\hline PINST & 101 & 1.917956 & 12.41444 & 0 & 88.81 \\
\hline MBA & 101 & 1.178743 & 1.111761 & 0.089 & 6.302 \\
\hline MBE & 101 & 5.154713 & 4.490102 & 0.794 & 25.8 \\
\hline INDCAUD & 101 & 0.3341564 & 0.4280925 & 0 & 1 \\
\hline ROA & 101 & 0.0442574 & 0.0700312 & -0.255 & 0.213 \\
\hline TAILLE & 101 & 17.88238 & 0.9380196 & 16.639 & 21.131 \\
\hline
\end{tabular}

Concernant la variable END, sa moyenne pour les firmes contrôlées par un «Big4» $(8,5 \%)$ est inférieure à celle qui sont auditées par les «non Big4» (10,07\%). Ceci montre que, dans le contexte tunisien, le niveau de l'endettement des firmes auditées par les «Big4» est inférieur à celui des autres firmes. Ce résultat implique que les firmes les moins endettées choisissent les cabinets d'audit de meilleure qualité. Ceci montre le caractère prudentiel des styles de gestion des entreprises tunisiennes. 
En outre, nous remarquons que les firmes auditées par un «Big4 » ont une moyenne de PINST supérieures à celle des autres firmes $(24,78 \%>1,918 \%)$. Ainsi, les firmes ayant plus d'investisseurs institutionnels choisissent un auditeur externe de meilleure qualité.

Concernant la moyenne de la variable MBA pour les firmes auditées par un «Big4 », celle-ci est supérieure à celle des autres firmes $(1,412>1,179)$. La même constatation est valable pour la moyenne de la variable $\mathbf{M B E}$ pour les firmes auditées par un «Big4». Elle est supérieure à celle des firmes auditées par les «Non Big4 ». Ceci implique que les firmes ayant de fortes opportunités d'investissement sont incitées à choisir un auditeur externe de meilleure qualité.

En ce qui concerne la variable INDCAUD, les firmes auditées par un «Big4 » se composent plus de membres indépendants que les autres firmes. En moyenne, 36,9\% des administrateurs du comité d'audit sont des membres indépendants dans les sociétés contrôlées par les «Big4», par rapport à 33,41\% de membres indépendants composant les comités d'audit des autres sociétés. Ce résultat n'est pas conforme au code de bonne gouvernance puisque ce pourcentage n'a pas atteint le pourcentage recommandé par ce code. De même, la moyenne de la variable ROA pour les entreprises qui sont contrôlées par un «Big4 » est supérieure à celle des autres entreprises (Non Big4), ceci montre que les firmes les plus rentables et les plus performantes sont incitées à choisir un auditeur externe de meilleure qualité.

\section{Interprétation des résultats :-}

Après avoir appliqué la matrice de corrélation de Pearson de l'ensemble des variables utilisées au niveau de notre premier modèle, nous avons examiné la multicolinéarité des variables explicatives en ayant recours à l'estimation Variance Inflation Factor (VIF). Le résultat dégagé de ce test ${ }^{1}$ montre que notre modèle ne pose pas de problèmes de multicolinéarité.

Nous rappelons que notre premier modèle à tester est le suivant :

Modèle 1:

Choix $_{i, t}=\propto_{0}+\propto_{1}$ MOD $_{i, t}+\propto_{2}$ END $_{i, t}+\propto_{3}$ PINST $_{i, t}+\propto_{4}$ MBA $_{i, t}+\propto_{5}$ INDCAUD $_{i, t}+\propto_{6}$

$\boldsymbol{R O} A_{i, t}+\propto_{7} T A I L L E_{i, t}+\varepsilon_{i, t}$

Le tableau 3 récapitule les résultats des estimations de la relation entre les caractéristiques de l'entreprise et le choix de l'auditeur.

Tableau 3 :- Résultats de l'estimation de la régression logistique du modèle (1)

Ce tableau présente les résultats de la régression logistique Choix ${ }_{i t t}=\alpha_{0}+\alpha_{1} \mathrm{MOD}_{\mathrm{it}}+\alpha_{2} \mathrm{END}_{\mathrm{it}}+\alpha_{3} \mathrm{PINST}_{\mathrm{it}}+\alpha_{4}$ MBA $+\alpha_{5}$ INDCAUD $_{\text {it }}+\alpha_{6}$ ROA $_{\text {it }}+\alpha_{7}$ TAILLE $_{\text {it }}+\varepsilon_{\text {it }}$ MOD est un attribut de la complexité opérationnelle et informationnelle. END présente le ratio d'endettement à long terme de la firme. PINST est la participation institutionnelle. MBA est le market to book asset, INDCAUD est l'indépendance du comité d'audit. ROA mesure la rentabilité de la firme. TAILLE est la taille de la firme. + désigne une relation positive, - désigne une relation négative, 0 désigne une relation non significative. *significatif au seuil de 1\%, *** significatif au seuil de $10 \%$.

\begin{tabular}{|l|l|l|l|l|l|l|l|}
\hline Variables & Coefficient & Std.Err. & \multicolumn{1}{|c|}{$\mathbf{Z}$} & $\begin{array}{c}\text { Signification } \\
\mathbf{P}>|\mathbf{Z}|\end{array}$ & $\begin{array}{c}\text { Signe } \\
\text { anticipé }\end{array}$ & $\begin{array}{c}\text { Signe } \\
\text { obtenu }\end{array}$ & Hypothèses \\
\hline MOD & -4.031177 & 1.494261 & -2.70 & $0.007^{*}$ & + & - & H1: infirmée \\
\hline END & -4.430219 & 2.346502 & -1.89 & $0.059^{* * *}$ & + & - & H2: infirmée \\
\hline PINST & -0.0451469 & 0.0694849 & -0.65 & 0.516 & + & 0 & H3: infirmée \\
\hline MBA & 0.3895444 & 0.2226192 & 1.75 & $0.080^{* * *}$ & + & + & H4: confirmée \\
\hline INDCAUD & -0.6203857 & 0.5434565 & -1.14 & 0.254 & + & 0 & H5 : infirmée \\
\hline TAILLE & 0.8803538 & 0.2641954 & 3.33 & $0.001^{*}$ & + & + & \\
\hline ROA & 0.9145337 & 2.850057 & 0.32 & 0.748 & + & 0 & \\
\hline CONS. & -15.84237 & 4.68081 & -3.38 & $0.001^{*}$ & & & \\
\hline \multicolumn{7}{|c|}{$\mathrm{R}^{2}=0.1748$} \\
\hline \multicolumn{7}{|c|}{ L.Rhi2 (7)=33.12 } \\
\hline \multicolumn{7}{|c|}{ Nombre d'observations = 150 } \\
\hline
\end{tabular}

${ }^{1}$ Les résultats sont disponibles sur demande. 
Le tableau 3 dévoile un Khi2 s'élevant à 33,12 ( $\mathrm{P}=0,000<0,01)$, ceci signifie que notre modèle est globalement significatif à $99 \%$. De même le $\mathrm{R}^{2}$ de notre modèle est égal à 0,1748 , ce qui nous permet de conclure que les variables utilisées expliquent à l'ordre de $17,48 \%$ le choix de l'auditeur. Les résultats de notre première régression dégagent un coefficient de la variable PINST non significatif. Celle-ci n'a pas d'effet sur le choix de l'auditeur externe de meilleure qualité. Ce résultat ne corrobore pas les aboutissements des études antérieures (Abdullah, 2008), Azibi et al., 2011 et Lajmi et Gana, 2012). Certes, les investisseurs institutionnels sont des acteurs importants sur le marché financier et occupent une place de plus en plus importante dans l'entreprise, mais ils n'ont aucun impact sur la demande de la qualité de l'audit approchée par la réputation de l'auditeur. Ceci nous amène à rejeter l'hypothèse H3. De tels investisseurs possèdent des compétences et des ressources nécessaires pour traiter l'information, ce qui leur permet d'exercer leur contrôle sur les dirigeants à moindre coût et n'ont pas, par ailleurs, le besoin de recourir à un audit externe de meilleure qualité.

En outre, pour ce qui est de la variable INDCAUD, nous observons que le coefficient de cette variable n'est pas significatif. Ce résultat montre que l'indépendance du comité d'audit n'influence pas la décision du choix des auditeurs externes pour les entreprises tunisiennes. Par conséquent, l'hypothèse H5 est rejetée. Ceci peut être expliqué par le fait que les administrateurs indépendants, considérés comme des contrôleurs efficaces, sont censés agir dans l'intérêt de l'entreprise et n'ont pas besoin de recourir à un audit externe de meilleure qualité. Ce résultat ne rejoint pas ceux obtenus par Broye (2009) et Lajmi et Gana (2013b).

Concernant la complexité opérationnelle et informationnelle des entreprises tunisiennes, celle-ci a un impact négatif et significatif au seuil de $1 \%$ sur la décision de choisir un auditeur externe appartenant à l'un des «Big4». Ce résultat n'est pas conforme à nos prévisions et aux résultats trouvés par Hay et Davis (2004). Nous rejetons alors notre hypothèse $\mathrm{H} 1$.

De plus, nous remarquons que la variable END a un effet négatif et significatif à un seuil de $10 \%$ sur la qualité de l'audit. Ce résultat observé n'est pas conforme à nos prévisions et aux recherches antérieures notamment celle de Hay et Davis (2004). Ces derniers montrent que les firmes les plus endettées ont tendance à choisir un auditeur externe de meilleure qualité appartenant aux grands cabinets d'audit «Big4». Ce qui nous amène à rejeter l'hypothèse $\mathrm{H} 2$.

Par ailleurs, les opportunités d'investissement MBA d'une firme ont un impact positif et significatif sur la sélection d'un auditeur externe de bonne qualité. Ce résultat est similaire à celui obtenu par Saghafi et Motamedi (2011). En effet, les entreprises ayant de fortes opportunités d'investissement, favorisent le choix d'un auditeur externe de meilleure qualité. Ceci nous amène à accepter notre hypothèse $\mathrm{H} 4$. Ce résultat peut être expliqué par le fait que les auditeurs appartenant au réseau «Big », sont susceptibles d'offrir un contrôle de qualité aux entreprises présentant des opportunités d'investissements élevés, afin de protéger leur capital de réputation en ayant moins des accruals discrétionnaires.

Pour ce qui est des variables de contrôle, les résultats de la régression affichés dans le tableau 3 montrent que la qualité de l'audit externe est influencée positivement par la taille de la firme. En ce sens, une relation positive et significative à un seuil de $1 \%$ entre les variables TAILLE et CHOIX $(\mathrm{p}=0,001)$ est dégagée. Ce résultat indique que les dirigeants des entreprises tunisiennes de grande taille optent pour un audit externe de meilleure qualité afin, de montrer qu'ils ne sont pas enracinés de manière négative.

Enfin, le coefficient de la variable ROA n'est pas significatif, ce qui implique que le niveau de la rentabilité de l'entreprise n'a pas une incidence sur le choix d'un auditeur externe de meilleure qualité. Notre résultat n'est pas compatible avec la théorie du signal selon laquelle les firmes les plus rentables signalent leur performance en ayant recours à une meilleure qualité d'audit.

Analyses de sensibilité :-

Modèle 2 :-

Choix $_{i, t}=\propto_{0}+\propto_{1}$ CTR $_{i, t}+\propto_{2}$ END $_{i, t}+\propto_{3}$ PINST $_{i, t}+\propto_{4}$ MBA $_{i, t}+\propto_{5}$ INDCAUD ${ }_{i, t}+\propto_{6}$ $\operatorname{ROA}_{i, t} \propto_{7} T A I L L E_{i, t}+\varepsilon_{i, t}$

Avec : 
CTR est un attribut de la complexité des transactions de l'entreprise, mesuré par le ratio suivant (Stocks + Créances + Produits à recevoir / Total Actifs).

Toutes les autres variables ont été expliquées dans le modèle (1).

Nous avons changé la mesure de la complexité de l'organisation par la CTR qui est la complexité des transactions de l'entreprise pour tester leur degré de sensibilité. Les résultats issus du modèle 2 sont présentés dans le tableau 4.

Tableau 4 :- Résultats de l'estimation de la régression logistique du modèle (2)

Ce tableau présente les résultats de la regression logistique Choix ${ }_{i t t}=\alpha_{0}+\alpha_{1}$ CTR $_{i t}+\alpha_{2}$ END $_{\text {it }}+\alpha_{3}$ PINST $_{\text {it }}+\alpha_{4}$ MBA $+\alpha_{5}$ INDCAUD $_{i t}+\alpha_{6}$ ROA $_{\text {it }}+\alpha_{7}$ TAILLE $_{i t}+\varepsilon_{i t}$ avec: CTR est un attribut de la complexité transactionnelle. END présente le ratio d'endettement à long terme de la firme. PINST est la participation institutionnelle. MBA est le market to book asset, INDCAUD est l'indépendance du comité d'audit. ROA mesure la rentabilité de la firme. TAILLE est la taille de la firme. + désigne une relation positive, - désigne une relation négative, 0 désigne une relation non significative. *significatif au seuil de $1 \%$, ** significatif au seuil de $5 \%$.

\begin{tabular}{|l|l|l|l|l|l|l|l|}
\hline Variables & Coefficient & Std.Err. & \multicolumn{1}{|c|}{$\mathbf{Z}$} & $\begin{array}{c}\text { Signification } \\
\mathbf{P}>|\mathbf{Z}|\end{array}$ & $\begin{array}{c}\text { Signe } \\
\text { anticipé }\end{array}$ & $\begin{array}{c}\text { Signe } \\
\text { obtenu }\end{array}$ & Hypothèses \\
\hline CTR & 3.552997 & 1.359903 & 2.61 & $0.009^{*}$ & + & + & H1 : confirmée \\
\hline END & -5.020753 & 2.336702 & -2.15 & $0.032^{* *}$ & + & - & H2 : infirmée \\
\hline PINST & -0.0345122 & 0.0737388 & -0.47 & 0.640 & + & 0 & H3 : infirmée \\
\hline MBA & 0.1225554 & 0.1887165 & 0.65 & 0.516 & + & 0 & H4 : infirmée \\
\hline INDCAUD & -0.4433341 & 0.5006127 & -0.89 & 0.376 & + & 0 & H5 : infirmée \\
\hline TAILLE & 1.397724 & 0.3303895 & 4.23 & $0.000^{*}$ & + & + & \\
\hline ROA & 2.506868 & 2.740862 & 0.91 & 0.360 & + & 0 & \\
\hline Cons & -27.46033 & 6.398978 & -4.29 & $0.000^{*}$ \\
\hline \multicolumn{7}{|c|}{ L.RKhi2 (7)=28.73 } \\
\hline \multicolumn{7}{|c|}{ Nobabilité > Khi2 $=0.0002$} \\
\hline
\end{tabular}

Nous remarquons que la variable CTR a un impact positif et significatif au seuil de $1 \%$ sur la décision du choix de l'auditeur externe appartenant aux cabinets «Big4». Le même résultat affiché dans le tableau 3 pour la variable END, est trouvé dans le tableau 4. Ce résultat change au niveau de la signification, c'est-à-dire que la variable END affecte négativement le choix d'un auditeur externe de bonne qualité, le coefficient de la variable END est négatif et significatif au seuil de 5\% au lieu du seuil de 10\% dans le tableau 4.

Nous constatons que la variable PINST n'est pas significative. Ce résultat confirme celui constaté dans le tableau 3.

En outre, nous observons que la variable MBA n'est pas significative. Ceci implique que les opportunités d'investissement n'ont pas un impact sur la décision du choix d'un auditeur externe de meilleure qualité. Ce résultat est différent à celui obtenu dans le tableau 3.

En ce qui concerne la variable INDCAUD, le résultat dégage un coefficient non significatif, confirmant ainsi celui trouvé dans le modèle précédent.

En analysant les variables de contrôle de notre modèle, nous constatons que les résultats relatifs aux variables TAILLE et ROA sont conformes à ceux trouvés dans le tableau 3.

Modèle 3 :-

$$
\begin{aligned}
& \text { Choix }_{i, t}=\propto_{0}+\propto_{1} \text { MOD }_{i, t}+\propto_{2} \text { END }_{i, t}+\propto_{3} \text { PINST }_{i, t}+\propto_{4} \text { MBE }_{i, t}+\propto_{5} \text { INDCAUD }_{i, t^{+}} \propto_{6} \\
& \text { ROA }_{i, t}+\propto_{7} \text { TAILLE } \\
& \text { Ti,t } \\
& +\varepsilon_{i, t}
\end{aligned}
$$

Avec : 
MBE : market to book equity, mesurée par le rapport entre la valeur de marché des capitaux propres et la valeur comptable des capitaux propres. Ici, nous remplaçons la variable MBA, qui reflète les opportunités d'investissement, par cette nouvelle variable MBE.

Les autres variables sont expliquées dans le modèle 1 .

Tableau 5 :- Résultats de l'estimation de la régression logistique du modèle (3)

Ce tableau présente les résultats de la regression logistique Choix ${ }_{i t t}=\alpha_{0}+\alpha_{1}$ MOD $_{\text {it }}+\alpha_{2}$ END $_{\text {it }}+\alpha_{3}$ PINST $_{\text {it }}+\alpha_{4}$ MBE $+\alpha_{5}$ INDCAUD $_{\text {it }}+\alpha_{6}$ ROA $_{i t}+\alpha_{7}$ TAILLE $_{\text {it }}+\varepsilon_{\text {it }}$ avec: MOD est un attribut de la complexité informationnelle et opérationnelle. END présente le ratio d'endettement à long terme de la firme. PINST est la participation institutionnelle. MBE est le market to book equity, INDCAUD est l'indépendance du comité d'audit. ROA mesure la rentabilité de la firme. TAILLE est la taille de la firme. + désigne une relation positive, - désigne une relation négative, 0 désigne une relation non significative. *significatif au seuil de 1\%, ** significatif au seuil de 5\%.

\begin{tabular}{|c|l|l|l|l|l|l|l|}
\hline Variables & Coefficient & Std.Err. & \multicolumn{1}{|l|}{$\mathbf{Z}$} & $\begin{array}{c}\text { Signification } \\
\mathbf{P}>|\mathbf{Z}|\end{array}$ & $\begin{array}{c}\text { Signe } \\
\text { anticipé }\end{array}$ & $\begin{array}{c}\text { Signe } \\
\text { obtenu }\end{array}$ & Hypothèses \\
\hline MOD & -3.308966 & 1.350711 & -2.45 & $0.014 * *$ & + & - & H1 : infirmée \\
\hline END & -5.953614 & 2.404458 & -2.48 & $0.013^{* *}$ & + & - & H2 : infirmée \\
\hline PINST & -0.0434169 & 0.075379 & -0.58 & 0.565 & + & 0 & H3 : infirmée \\
\hline MBE & 0.0498442 & 0.0369856 & 1.35 & 0.178 & + & 0 & H4 : infirmée \\
\hline INDCAUD & -0.6283565 & 0.5320389 & -1.18 & 0.238 & + & 0 & H5 : infirmée \\
\hline TAILLE & 0.8267648 & 0.2647314 & 3.12 & $0.002^{*}$ & + & + & \\
\hline ROA & 1.622319 & 2.782159 & 0.58 & 0.560 & + & 0 & \\
\hline Cons & -14.69297 & 4.677938 & -3.14 & $0.002^{*}$ & & & \\
\hline \multicolumn{7}{|c|}{ L.RKhi2 (7)=31.85 } \\
\hline \multicolumn{7}{|c|}{ Nobabilité > Khi2 $=0.0000$} \\
\hline \multicolumn{7}{|c|}{ Nombre d'observations = 150 } \\
\hline
\end{tabular}

Les résultats issus du tableau 5 dévoilent que la variable MOD influence négativement et significativement à un seuil de 5\% la décision du choix d'un auditeur externe de bonne qualité au lieu du seuil de $1 \%$ dans le tableau 3 .

Pour ce qui est de l'endettement de l'entreprise, cette variable présente un impact négatif et statistiquement significatif au seuil de 5\% sur la décision du choix d'un auditeur externe de meilleure qualité, au lieu du seuil de $10 \%$ dans le tableau 3.

En outre, la propriété institutionnelle n'est pas significative. Ce résultat est similaire à celui trouvé dans le tableau 3.

De même, nous constatons que la variable MBE n'est pas significative c'est-à-dire que les opportunités d'investissement de l'entreprise n'ont pas une incidence sur la décision de choix d'un auditeur externe de meilleure qualité.

De plus, la variable INDCAUD n'est pas significative. Ce résultat est concordant à celui dégagé dans le tableau 3 de la régression logistique du premier modèle.

Enfin, les résultats issus du tableau 5 concernant les variables TAILLE et ROA, sont conformes à ceux obtenus par le premier modèle.

De cette dernière régression, nous pouvons constater que les résultats ainsi dégagés sont relativement concordants à ceux obtenus par le premier modèle. Par conséquent, nous pouvons conclure que notre modèle initial est robuste.

\section{Conclusion :-}

L'objet de cette étude était de tester empiriquement l'impact de certaines caractéristiques organisationnelles et de gouvernance sur la décision du choix des auditeurs externes. Pour ce faire, nous avons identifié les auditeurs externes de bonne qualité en Tunisie par leur appartenance à l'un des réseaux internationaux «Big4 ». Afin de répondre à notre problématique, nous avons eu recours à la régression logistique, en se basant sur l'économétrie des données de panel de 25 entreprises observées sur une période de 6 ans. Les résultats ont montré que les grandes 
firmes ayant de fortes opportunités d'investissement préfèrent choisir un auditeur externe appartenant au groupe «Big4 ».

Pour ce qui est de la variable «main d'œuvre direct» et la variable « endettement», celles-ci ont une incidence plutôt négative et significative sur la décision du choix de l'auditeur externe. Ceci penche en faveur d'un effet de substitution.

Enfin, les variables «propriété institutionnelle», «indépendance du comité d'audit» et «rentabilité de l'entreprise » n'ont relevé aucun impact sur la décision du choix d'un auditeur externe de meilleure qualité.

Ainsi, les résultats de notre recherche améliorent notre compréhension du comportement des sociétés tunisiennes dans le choix de leurs auditeurs externes. Ceci est de nature à aider les dirigeants à choisir les auditeurs les mieux réputés sur le marché en fonction des caractéristiques de leurs entreprises. Notre travail participe, par ailleurs, au débat actuel sur le choix d'un audit de meilleure qualité.

Toutefois, malgré la significativité de certaines relations examinées, l'ajout d'autres variables justifiant le choix des auditeurs au sein des firmes tunisiennes, pourrait améliorer notre étude. Par ailleurs, d'autres caractéristiques de gouvernance intégrées dans l'étude, notamment l'indépendance et la taille du conseil d'administration, l'expertise et la diligence du comité d'audit, pourraient faire l'objet de futures recherches. D'autres mesures de la qualité de l'audit pourraient, par ailleurs, être adoptées afin de construire un indice composite pour améliorer la recherche empirique.

\section{References:-}

1. Abdullah, W.Z.W. (2008). The Impact of Board Composition, Ownership and CEO Duality on Audit Quality: The Malaysian Evidence. Malaysian Accounting Review, 7 (2), 17-28.

2. Azibi, J.,T., Ondeur H. \& Rajhi M.T. (2011). Auditor Choice and Institutional Investor Characteristics after The Enron Scandal in The French Context. International Journal of Economics and Accounting, 2 (1), 32-52.

3. Azibi, J. (2014). Qualité d'Audit, Comité d'Audit et Crédibilité des Etats Financiers après le Scandale Enron : Approche Empirique dans le Contexte Français, Thèse de doctorat.

4. Broye, G. (2009). Honoraires d'Audit et Comité d'Audit: le Cas de la France. Comptabilité, Contrôle, Audit, 1 (15), 199-224.

5. Charléty, P. (2001). Le Gouvernement d'Entreprise : Evolution en France Depuis le Rapport Viénot de 1995. Revue d'Economie Financière, 63 (3), 25-34.

6. Choi, J-H. \& Lee, W-J. (2014). Association Between Big 4 Auditor Choice and Cost of Equity Capital for Multiple-Segment Firms. Accounting and Finance, 54 (1), 135-163.

7. Craswell, A.T., Stokes, D.J. \& Laughton J. (2002). Auditor Independence and Fee Dependence. Journal of Accounting and Economics, 33 (2), 253-275.

8. Dumontier, P., Chtourou, S. \& Ayedi S. (2006). La Qualité de l'Audit Externe et les Mécanismes de Gouvernance des Entreprises: Une Etude Empirique Menée dans le Contexte Tunisien. Actes du 27ème congrès de l'Association Francophone de Comptabilité (AFC), Tunis.

9. Fama, E.F. \& Jensen, M.C. (1983). Agency Problems and Residual Claims. Journal of Law and Economics, 26, 327-349.

10. Gul, F.A. \& Tsui, J.S.L. (1998). A Test of The Free Cash Flow and Debt Minorting Hypothesis: Evidence From Audit Pricing. Journal of Accounting and Economics, 24, 219-237.

11. Hay, D. \& Davis, D. (2004). The Voluntary Choice of an Audit of any Level of Quality. Auditing: A Journal of Practice and Theory, 23 (2), 37-53.

12. Hay, D., Knechel, W.R. \& Wong, N. (2006). Audit Fees: a Meta-Analysis of the Effect of Supply and Demand Attributes. Contemporary Accounting Research, 23, 141-192.

13. Hsu, W.Y., Troy, C. \& Huang, Y.R. (2015). The Determinants of Auditor Choice and Audit Pricing Among Pr operty-Liability insurers. J. Account. Public Policy, 34, 95-124.

14. Jensen, M. C. \& Meckling, W. H. (1976). Theory of the firm: managerial behavior, agency costs and ownership structure. Journal of Financial Economics, 3, 305-360.

15. Joshi, P.L. \& AL-Bastaki, H. (2000). Determinants of Audit Fees: Evidence From The Companies Listed in Bahrain. International Journal of Auditing, 4 (2), 129-138.

16. Kane, G.D.; Velury, U. (2005). The Impact of Managerial Ownership on the Likelihood of Provision of High Quality Auditing Services. Review of Accounting and Finance, 4 (2), 86-106. 
17. Lai, K.W. (2009). Does Audit Quality Matter more for Firms with High Investment Opportunities ? J. Account. Public Policy, 28, 33-50.

18. Lajmi, A. \& Gana, M. (2012). Structure de Propriété et Qualité de l'Audit Externe dans les Entreprises Belges Cotées. Gestion 2000, Bimestriel 3/2012, 29, 83-96.

19. Lajmi, A. \& Gana, M. (2013a). Board Characteristics and External Audit Quality: Complementary or Substitute Mechanisms? The Belgium Case. International Journal of Management and Business Research, 2 (3), 68-74.

20. Lajmi, A. \& Gana, M. (2013b). Audit Committee and External Audit Quality: Substitution or Complementarity? Empirical Evidence on Euronext Brussels. International Journal of Financial Management Research and Development, PRJ Publication, 3 (2), 22-38.

21. Lang, L.H.P. \& Litzenberger, R. (1989). Dividend Announcements: Cash Flow Signaling vs. Free Cash Flow Hypothesis. Journal of Financial Economics, 24, 181-192.

22. Miko, N.U. \& Kamardin, H. (2015). Impact of Audit Committee and Audit Quality on Preventing Earning Management in the Pre- and Post- Nigerian Corporate Governance Code 2011. Procedia- Social Behavior Sciences, 172, 651-657.

23. Nekhili, M., Masmoudi, W. \& Nekhili, D. (2009). Choix de l'Auditeur Externe, Honoraires d'Audit et Gouvernance des Entreprises Françaises. Cahier du FARGO n 1090501 , Université de Bourgogne.

24. Omri, M.A. \& Dallai, H. (2013). Déterminants du choix des auditeurs externes dans le context tunisien, Recherche en Sciences de Gestion,2 (95), 131-148.

25. Palmorse, Z. (1988). An Analysis of Auditor Litigation and Audit Service Quality. The Accounting Review, 63 (1), 55-73.

26. Saghafi, A. \& Motamedi, M. (2011). Relationship Between Audit Quality and Investment Efficiency in Firms with High Investment Opportunities. Journal of Financial Accounting research, 3 (4), 1-14.

27. Skinner, D.J. (1993). The Investment Opportunity Set and Accounting Procedure Choice: Preliminary Evidence. Journal of Financial Economics, 16, 407-445.

28. Smith, C.W. \& Watts, R.L. (1992). The Investment Opportunity Set and Corporate Financing, Dividend and Compensation Policies. Journal of Financial Economics, 32, 263-292. 